\title{
STUDYING THE RELATIONSHIP OF LOCALIZATION PARAMETERS OF SOLAR SOURCES OF MAGNETIC CLOUDS WITH THEIR CHARACTERISTICS AND SUBSTORM ACTIVITY
}

\author{
N.A. Barkhatov \\ Minin Nizhny Novgorod State Pedagogical University, \\ Nizhny Novgorod, Russia,nbarkhatov@inbox.ru
}

\section{E.A. Revunova}

Nizhny Novgorod State University of Architecture and Civil Engineering, Nizhny Novgorod, Russia,

revunova.elena@mail.ru

\section{R.V. Romanov}

Minin Nizhny Novgorod State Pedagogical University,Nizhny Novgorod,Russia,r-o-m-e-n@yandex.ru

\section{O.M. Barkhatova}

Nizhny Novgorod State University of Architecture and Civil Engineering, Nizhny Novgorod, Russia, o.barkhatova@inbox.ru

\author{
S.E Revunov \\ Minin Nizhny Novgorod State Pedagogical University, \\ Nizhny Novgorod, Russia, revunov@inbox.ru
}

\begin{abstract}
We propose a method for determining location and orientation of extended solar sources of magnetic clouds, using coronagraph data and $\mathrm{SOHO}$ EIT/MDI images of the photosphere. To estimate the probability of formation of magnetic clouds, we use a simple cylindrical force-free model. We have established that more extended sources having a slight inclination to the solar equator and located on the solar limb as compared to those that are nonextended and strongly inclined can generate expanding clouds, which with high probability can reach the magnetosphere as well as clouds from a source near the zero meridian and low latitudes.

We have determined the relationship between extreme values of substorm activity and parameters of
\end{abstract}

solar sources under study during the impact of magnetic clouds on Earth's magnetosphere from the AL index. The absence of substorms associated with extended sources outside the heliolatitude range $\sim 5-20^{\circ}$ is noted. The established relationship between solar source coordinates and geomagnetic activity of the magnetic cloud sheath and body are consistent with the most probable distribution of magnetoactive regions over the solar disk.

Keywords: solar activity, solar wind, coronal plasma flow, coronal mass ejection, solar flare, geomagnetic activity, geomagnetic disturbances, magnetosphere.

\section{INTRODUCTION}

Coronal mass ejections (CMEs) and flares often accompanying them are the highest-energy solar events [Ivanov, 1996; Burlaga et al., 2003; Neugebauer, Liewer, 2003; Nikolaeva et al., 2011]. While CME occurs with the release of large amount of energy, flares, and microwave bursts, CME sources often do not stand out in the solar corona or photosphere, and can sometimes be determined only from residual effects. Special attention should be given to $\mathrm{CME}$ of such type (or, rather, its attendant phenomenon) as magnetic cloud (MC). Unlike $\mathrm{CME}, \mathrm{MC}$ can have a huge amount of magnetic field energy practically without mechanical momentum [Kilpua et al., 2012]. Because of extremely low content of particles, MC is weakly exposed to solar gravity and can increase the specific magnetic field energy due to compression when interacting with other dense solar wind structures [Barkhatova et al., 2017; Manakova et al., 2016]. Analysis of MC and long-term forecast of its geomagnetic effectiveness are hampered by the fact that an isolated MC is rare and its source is difficult to detect against the background of the disturbed solar corona or photosphere. It is, however, assumed that the high level of CME geoeffectiveness results from low heliolatitudes of CME sources [Kilpua et al., 2011] and equatorial deflection of slow and weak CMEs originating from middle and high heliolatitudes [Liu et al., 2008].

Due to roughly northward orientation of the geomagnetic field in the dayside magnetosphere, it is logical to construct statistical dependence of geomagnetic effectiveness on the solar wind structures having a noticeable negative $B_{z}$ component of the interplanetary magnetic field (IMF) [Wu, Lepping, 2002; Barkhatov et al., 2017, 2018]. Indeed, explosive reconnections of the geomagnetic and interplanetary magnetic fields responsible for global geomagnetic storms occur only with the negative $B_{z}$ component of IMF.

Geomagnetic effectiveness of MC can be predicted from the negative $B_{z}$ component with an accuracy greater than $60 \%$. To improve the quality of the forecast requires data on MC sources and starting physical characteristics. It is also necessary to maximally accurately identify structures with MCs passing through patrol spacecraft (SC) [Riazantseva et al., 2003]. In recent years, space missions, such as TRACE, have centered on this.

Difficulties in identifying MCs are associated with the fact that coronagraphs record solar wind proton density 
perturbations caused by penetration of high-energy coronal protons into the static diffuse background of protons emitted by the Sun. In view of this, MCs even in profile are poorly seen in coronagraph images. Propagation of isolated MCs to Earth is not recorded by coronagraphs at all, and their likely sources can be identified only from images of the solar photosphere. Thus, in general there is a structure measured by patrol SC with a set of likely solar sources and disembodied data on the movement of this structure. The problem about parameters of solar structures when they move from the Sun to patrol SC is solved by numerical or, in the simplest cases, analytical simulation. It becomes easier if the number of coronographs increases, but the problem of identifying MCs from coronagraph data still remains unsolved. Our approach to $\mathrm{MC}$ simulation is that their magnetic part is a closed structure of force-free magnetic tube type. Its material is plasma with frozen-in magnetic field carried away from the Sun during eruption. Meanwhile, the halo CME body is readily observed by coronagraphs. This allows quite reliable identification of CME sources on the solar disk and CMEs by patrol SC [Wang et al., 2011]. In this case, such CMEs also include $\mathrm{MCs}$, therefore we can also identify an MC source. Our approach to finding CMEs, including MCs, and identifying their solar sources involves using the above capabilities.

Another problem of studying peculiarities of CME transfer is their huge size. Even the transverse size of CMEs can reach 1 AU. The hypothetical flotilla of patrol spacecraft in this case can be compared with a wire probe in the body of a whale, and from their measurements we cannot get even a plane cross-section of the structure under study. The solution of such problems therefore depends entirely on adequacy of models. Nowadays, the most common models are the MC models assuming their local cylindrical symmetry. Such models provide dynamics of fields in the plane crosssection of the structure. Given small scales of the geomagnetosphere as compared to CME, it suffices to compute parameters on the structure cross-section in the plane of the ecliptic and then to compare them with measurements from patrol SC and evaluate adequacy of the model. Such approach allows us to solve the inverse problem of determining model parameters of CME from measurements made by patrol SC. The obtained model parameters allow us to compare this CME structure with its solar source.

Taking into account all these problems, the proposed study is aimed at establishing the relationship between coordinates of solar regions of localization of plasma stream sources and substorm geomagnetic activity level. The developed approach to the study of the MC-type plasma stream represents its magnetic part as force-free magnetic flux tube carried away from the Sun during eruption [Barkhatov, Kalinina, 2010].

Parametric SC recordings often show the MC body and sometimes a shock wave (SW) and its associated turbulent sheath (TS) (see Figure 1). Therefore, despite the fact that clouds move in near-Earth space as a whole, we should consider the geomagnetic effectiveness of elements of their structure in isolation from one another. In TS, processes occur which are similar to those in the magnetosheath (transition region) behind a terrestrial shock wave. Power of magnetosonic pulsa- tions in the interplanetary coronal mass ejection (ICME) is by an order of magnitude lower than that of turbulent perturbations in MC sheath; however, owing to intermittency of turbulence it is difficult to use spectral methods for separating the sheath from the ICME body.

The comparison between existing MC models [Barkhatov, Kalinina, 2010; Barkhatov et al., 2014a, b] has shown that they all agree with each other and their complexity does not give significant insight into the main cloud parameters (the magnitude of the magnetic field on the cloud axis, its radius, impact parameter with respect to Earth, axis orientation in interplanetary space). To estimate the MC orientation, we therefore use a simple force-free cylindrical model (Figure 2).

\section{DATA AND METHOD
FOR LOCALIZING
MC SOLAR SOURCE}

The statistical study of relationships between MC source parameters and location on the solar disk is based on 30 MC events occurring from 1997 to 2012. From the following catalogs: 1) LASCO CME Catalog [http://lasco-www.nrl.na-vy.mil/index.php?p=content/ cmelist]; 2) large-scale solar wind events [https://cdaw.gsfc.nasa.gov/CME_list/]; 3) flares with indication of the presence of CMEs and shock waves

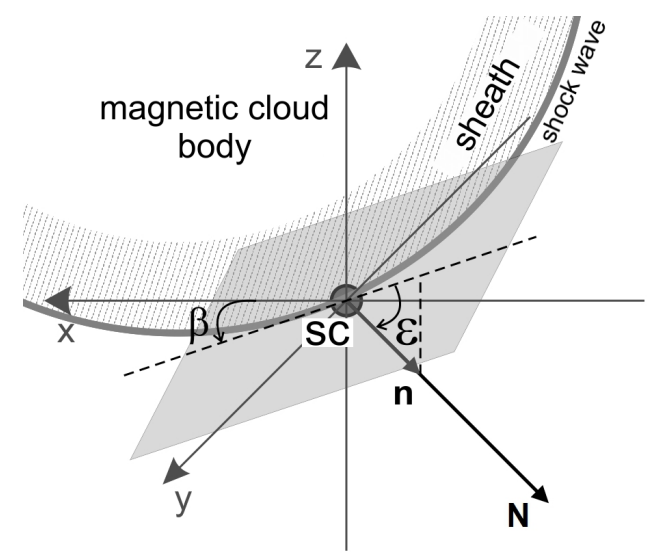

Figure 1. Magnetic cloud structure comprising a body, sheath, and shock wave (SW) with a conditioned plane front considered at the point of intersection with SC. Azimuthal $\beta$ and latitudinal $\varepsilon$ angles characterize the orientation of normal $n$ to the shock wave of MC in interplanetary space in the GSE coordinate system

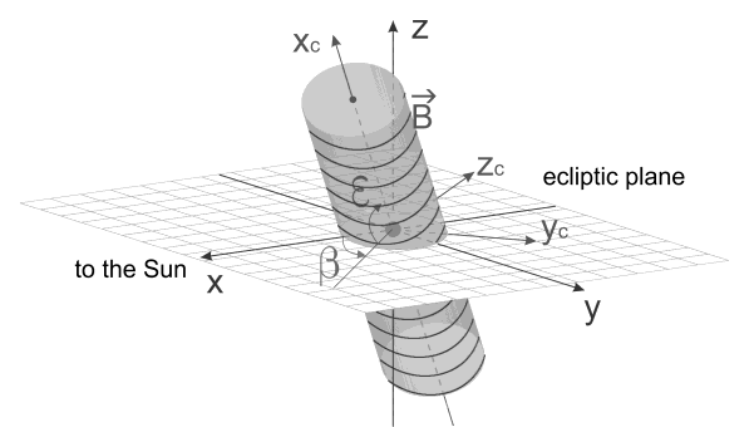

Figure 2. Orientation angles of MC represented by a force-free cylindrical magnetic flux tube [Barkhatov et al., 2009]. 
[http://umtof.umd.edu/sem/]; 4) $\mathrm{H} \alpha$ flares [ftp:// ftp.ngdc.noaa.gov/STP/SOLAR_DATA/]; 5) X-ray flares from the Virtual Solar Observatory [http://vso.nso.edu/cgi/catalogue], we have identified $\sim 70$ possible solar sources for MC.

The initial stage of MC solar source localization involves determining the number of likely sources per day, starting from the time that is calculated from the minimum velocity of CME propagation from the Sun to a patrol SC. The minimum velocity is taken to be equal to the sum of mean MC velocity (from SC data) and mean magnetic sound velocity (from SC data). MC sources are extended, therefore we consider their angular coordinates, the nearest to the center of the heliocentric Cartesian coordinate system. This approach seems to be the most convenient for studying the statistics associated with solar coordinates of sources, and better reflects their geoeffectiveness because the lower is the source in latitude, the greater part of CME arrives at near-Earth space. The same considerations apply to longitudes.

In general, it is difficult to determine CME source coordinates. First, many events occur with cascade eruptive processes involving loops from several regions. Second, in many cases, the coronagraph resolution is insufficient for observations of sunspots and eruptive processes. Third, structures of many sources are far from representing the flux loop as an arch (e.g., sigmoids).

In this study, we propose the following method for localizing solar sources. As MC sources we take regions of emergence and eruption of flux loops (filaments). Determination of their coordinates reduces to determining coordinates of visible regions of emergence of these structures in the photosphere (footpoints) from coronagraph data. There are almost no problems with highenergy events because loops make a contrast with the photosphere and sunspots are clearly visible. In the case of the cascade process, by a source is meant an area in which the final loop decays (footpoints of this loop) and becomes invisible in the frequency band of the coronagraph.

If the coronagraph cannot observe the eruption, we use indirect signs of eruption (such as post-eruptive arcades, deflection of field lines around the invisible center, darkening areas). The area of loop emergence is determined from arcades; and coordinates of footpoints, from deflections of field lines around the invisible cen- ter in areas where arcades end. The coordinates of sources differing from classical arched flux loops are useless for statistics because they do not reflect the scale of the event. In such cases, instead of point coordinates of footpoints we utilize values of latitudes and longitudes of boundaries of areas involved in the eruption.

In the period of high solar activity, the number of likely CME sources may exceed 10 per day. Therefore, the next criterion for selecting likely sources is the presence of X-ray flares and microwave bursts. It is believed that these phenomena in some cases are associated with CME [Hundhausen, 1999], and since they are described with 1-min resolution we can compare them with SO$\mathrm{HO} / \mathrm{EIT}$ images of the solar photosphere and determine flare coordinates. Many CMEs are, however, followed by flares of C-class and lower, which may not stand out against a background of X-ray emission of solar active regions at all. Moreover, we should bear in mind that matching of CME sources to coordinates of flares that accompany the eruption plays havoc with statistics because flares often occur in different areas of the same extended source.

The results of application of the proposed method for localizing MC sources are most easily checked using halo CMEs, which on SOHO LASCO images look like closed halos. For the halo ejected anti-earthward, we should use data on X-ray and microwave radiation. In successful cases, resolution of LASCO C2 is $24 \mathrm{~min}$, and it is sufficient for comparison with the likely source in SOHO/EIT images within an accessible range (usually $195 \AA$ ). Wherever possible, the obtained coordinates of $\mathrm{CME} / \mathrm{MC}$ sources were compared with coordinates of sunspots in the vicinity of the ejection source, using SOHO/MDI images (Figure 3,a). The coincidence with the deflection at most $2-3^{\circ}$ in latitude was considered satisfactory. In determining the source coordinates, we also took into account SC SOHO rocking about the GSE X-axis in a range of $\sim \pm 7.85^{\circ}$. Rocking angles for each case were calculated from the movement of stars in LASCO C3 differential images during the day or a slightly shorter period (Figure $3 b, c$ ).

In this case (Figure 3), the inclination of the solar equator is apparent. If we track the movement of stars from LASCO C3 images, we can find out that their daily track has an angle of $\sim-7.35^{\circ}$ to horizontal, i.e. the deri-
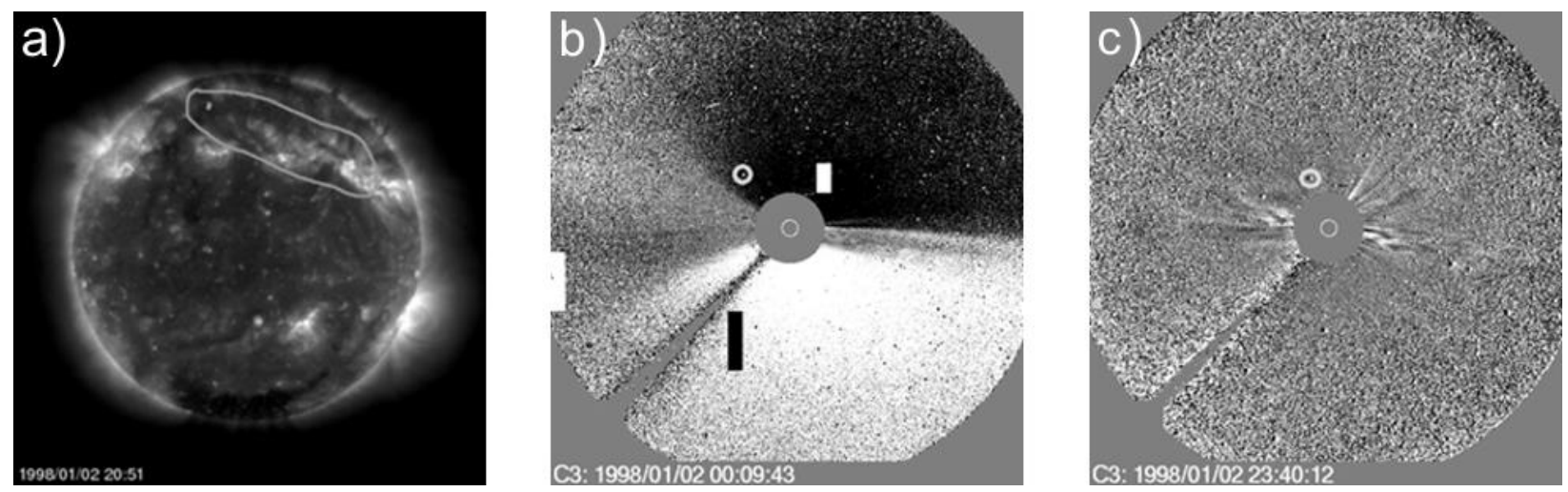

Figure 3. SOHO images used to determine coordinates of CME/MC sources: sunspot region in the vicinity of a CME source at a wavelength of $195 \AA$ (MDI) (a); CME, the circle marks a fixed star relative to which rocking angles (LASCO C3) are determined $(b, c)$ 
ved coordinates of sources need to be tightened further $\sim+7.35^{\circ}$. The particle density in CME is at least two times higher than that in the disturbed SW and ten times higher than that in the quiet SW. It is therefore easy to determine ICMEs from parameters measured by patrol SC. The studied MC and ICME it accompanies emerge from one source and have the same coordinates.

Using the obtained coordinates, we can also derive other parameters of sources: their angular size and inclination in the plane of the sky (as the source is seen by the coronagraph) in the Cartesian heliocentric coordinate system. We should expect that due to the differential rotation of the Sun MC sources are extended at acute angles to the heliographic equator: negative in the northern hemisphere and positive in the southern hemisphere.

When to one structure of interest correspond two sources of halo CMEs, we compare velocities of structures measured by patrol SC. The second CME should have a higher velocity to catch up with (and possibly overtake) the first one. We therefore determine the velocities of the corresponding medium consolidations identified with ICMEs, and set their priority in accordance with the sources. It has to be said that the situation when the second CME overtakes the first, slower one at a distance of $1 \mathrm{AU}$ is extremely rare even if there are only a few hours between onsets of the CMEs on the Sun.

It is also rare for the second, faster CME to penetrate through the first one. The second, faster CME more often catches up with the first one and presses it together with its attendant structures, i.e. the chronological sequence of monitoring of the structures, identified with ICME, usually corresponds to the chronological sequence of likely sources. In the case of two likely sources, the MC of interest is often clamped between two ejections and has two shock-wave transition regions - before and behind. In this case, the source of the second MC is the source of the first CME. Note that the attendant MC does not always follow the leading ICME - it can be within the ejection or even be ahead of it, with a slight medium consolidation, rather than a turbulent sheath, in front.

An important parameter of the structures measured by patrol SC is the magnetic flux through the flux tube cross-section (axial magnetic field). This flux is usually calculated on the basis of the model in use and consists of axial and azimuthal components (toroidal and poloidal for a torus model).

Our approach is to simplify the problem on the basis of physical considerations. In SW, magnetic structures are hardly ever free to move because of the relatively small mechanical momentum. They move in a compressed state due to deceleration by the leading ICME (because of the higher density it more rapidly loses speed due to the solar gravity) or due to pressure of another ICME moving with a higher velocity behind. When the magnetic flux tube is compressed, the azimuthal magnetic field decreases, and the axial one increases due to conservation of the first and second adiabatic invariants, i.e. the stronger the magnetic flux tube is compressed across the axis, the greater is the axial magnetic field component it contains. The orientation of the strongest magnetic field is the closest to the axial one.

\section{RELATIONSHIP OF PARAMETERS OF CME SOLAR SOURCES WITH THEMSELVES AND WITH MC CHARACTERISTICS}

Consider the relationship of localization and orientation parameters of CME solar sources with each other and with $\mathrm{MC}$ characteristics. In addition to CME solar source coordinates, the most objective data is that on the angular orientation of its visible part (filament, magnetic flux filament) on the solar sphere relative the heliographic equator, as well as on the source length measured in degrees. It is therefore worth examining the dependence of these two parameters upon source coordinates on the solar disk. In what follows, by source coordinates is meant the minimum width of the extended source and respective longitude. The dependence of parameters of MC structures measured by SC on solar source parameters and the relationship between these parameters were studied using a correlation analysis. We have found the following regularities.

\subsection{Relationship of angular orientation of the visible part of the solar source with its coordinates}

We have found that the closer is a source to the heliographic equator, the more extended is it in the equatorial direction, and vice versa the farther is the source from the equator, the more extended is it in the meridional direction. This is especially evident for northern and southern latitudes of the western hemisphere, where the minimum inclination of the source is observed at zero latitudes with a correlation coefficient $R=0.72$ (Figure 4). This is most likely to be due to the effect of the differential rotation of the photosphere on the source. The longitudinal dependence of the source inclination angle corresponding to the minimum latitude of the extended source is weak. Nevertheless, we can conclude that inclination angles of the extended source decrease with increasing latitude. The latter can be explained by features of magnetic cloud expansion, due to which MCs from "lying" sources (i.e. nearly parallel to the plane of the solar equator [Barkhatov, Kalinina, 2010]) from the

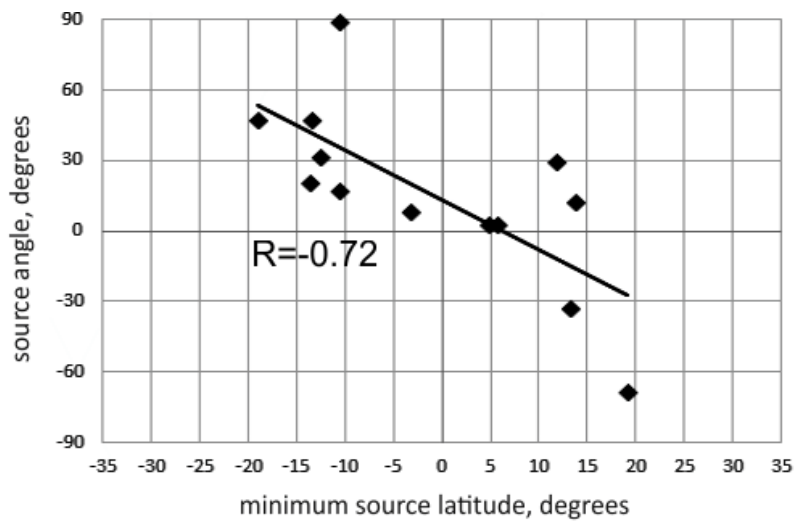

Figure 4. Inclination of an extended source to the solar equator versus the minimum latitude (in the western hemisphere) 
periphery of the solar disk expand mainly equatorward and might more likely affect near-Earth space than MCs from "standing" sources (i.e. elevated above the plane of the solar equator by more than $60^{\circ}$ ). MCs from regions near the zero meridian reach Earth's magnetosphere regardless of the orientation of the extended sources.

\subsection{Distribution of solar sources of different lengths}

There is a dependence of source length (measured in degrees) on its minimum latitude with minimum near the equator. This dependence is especially pronounced for the northeastern solar disk (Figure 5), being more pronounced in the northern hemisphere than in the southern one. Thus, as the latitude of MC source increases the probability of detection of $\mathrm{MC}$ in the plane of the ecliptic, where the patrol SC is located, decreases. It is, however, possible to record MCs in near-Earth space for the high-latitude extended sources generating larger MCs. The length of solar sources with the minimum latitude of the extended source near the noon meridian increases with longitude from a minimum value of $\sim 10^{\circ}$ both eastward and westward (Figure 6). Indeed, extended sources on solar limbs unlike nonextended ones have a chance to generate expanding MCs that can reach the magnetosphere.

2.3. Relationship of MC velocity with minimum latitude of extended sources and its associated longitude

For the northern hemisphere, there is a direct relationship $(\mathrm{R}>0.45)$ of $\mathrm{MC}$ velocity with latitude. It is noticeable

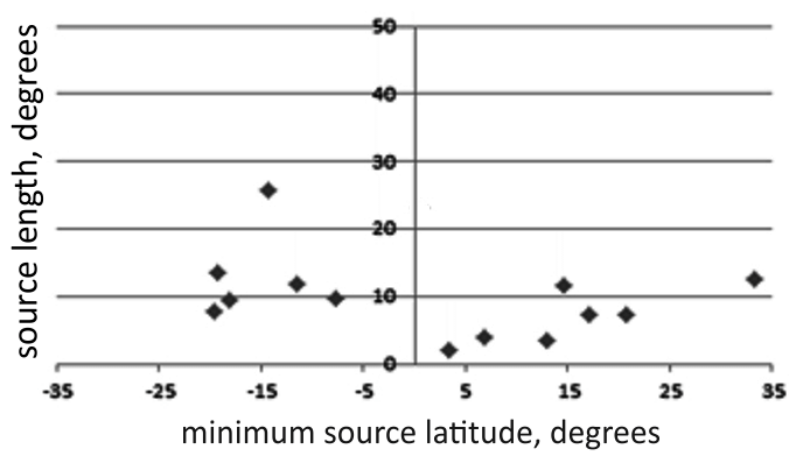

Figure 5. Distribution of source length over the latitude in the eastern solar disk

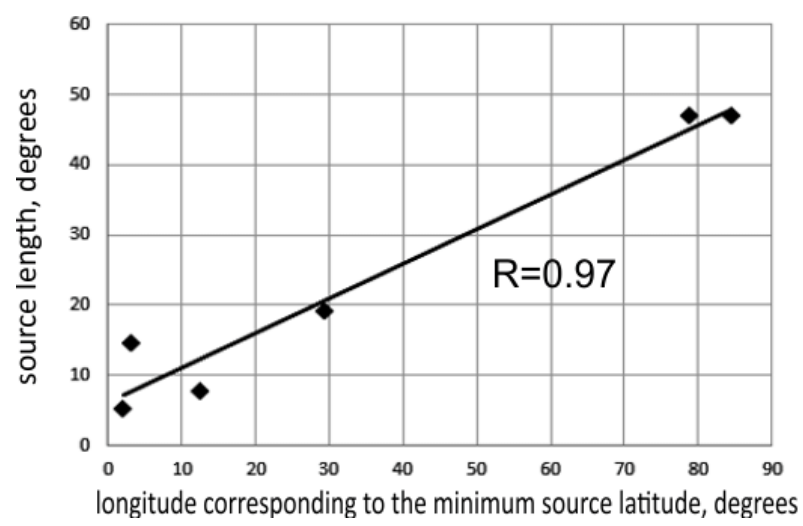

Figure 6. Source length as a function of longitude corresponding to the minimum latitude (for the western solar disk) that the more to the north and east is the likely source of the structure from the Sun, the higher is its velocity (see Figures 7,8).

The MC velocity dependence on the source latitude in the northern and southern hemispheres of the Sun per quadrant has a correlation coefficient $R$ from 0.5 to 0.7 . There is no mirror symmetry in this dependence for the southern hemisphere in our data.

Figure 8 shows the spatial distribution of $\mathrm{MC}$ sources over the solar disk illustrating the distribution of MC velocities. The observed increases in the number of high-speed MCs related to the eastern solar disk and the number of slow MCs related to the western one reflect effects of the spiral motion of the solar wind stream conditioned by the solar angular velocity. In the former case, the spiral is weaker and MCs are recorded closer to the Sun-Earth line.

\subsection{Relationship of maximum magnetic field} strength in MC with minimum latitude of extended source and its associated longitude

The distribution of maximum magnetic field (MF) strength in MC depending on the minimum latitude of an extended solar source exhibits values up to $\sim 50 \mathrm{nT}$ at 15 $20^{\circ}$ latitudes with minimum values of the order of $\sim 10 \mathrm{nT}$ near the heliographic equator (Figure 9, a). Maximum MF values in this case correspond to regions near the noon meridian (Figure 9, $b$ ). Thus, for a large group of magnetic structures there is a direct latitudinal dependence of their maximum magnetic field on source coordinates.

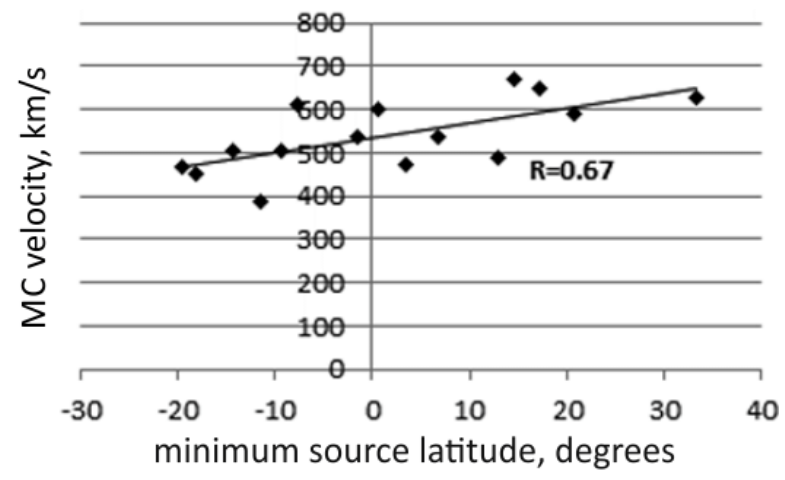

Figure 7. MC velocity versus minimum source latitude

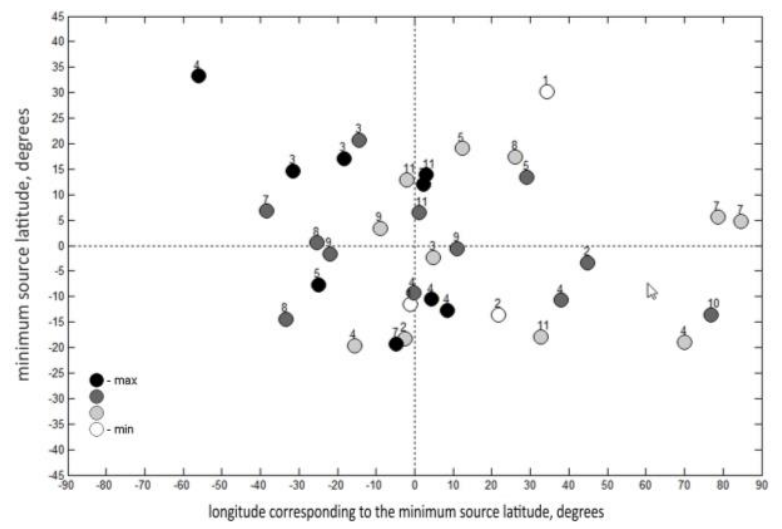

Figure 8. MC velocity versus minimum latitude and its associated longitude of the extended source. Color of markers (from white to black) represents velocities (from 400 to 600 $\mathrm{km} / \mathrm{s}$ and higher); the number indicates the month of recording for each event 


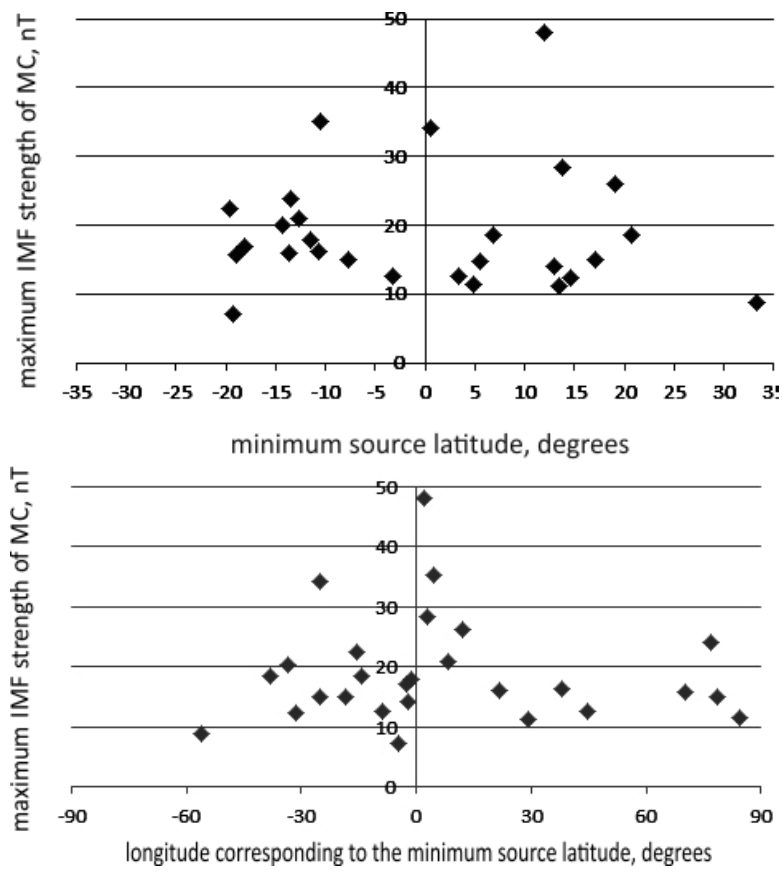

Figure 9. Distribution of the maximum magnetic field strength in MC depending on the minimum latitude $(a)$ and on the longitude corresponding to the minimum latitude of the source for both hemispheres $(b)$

This dependence with the minimum in the vicinity of the equator $(R \sim-0.6)$ is especially pronounced for the northeast quadrant. The maximum MF strength in MC increases with increasing northern and southern latitudes. In general, the maximum MF strength in MC has a weak negative relationship with longitude corresponding to the minimum latitude of the source. In the western hemisphere, the negative relationship of the maximum MF strength in $\mathrm{MC}$ with longitude of the source (to $R \sim-0.7$ ) is more pronounced.

2.5. Relationship of the $B z$ component of maximum MF strength in MC with minimum source latitude and its associated longitude

Relationships of the $B_{z}$ modulus of the maximum MF strength with the minimum latitude and its associated longitude of the solar source exhibit a minimum near the heliographic equator and a maximum ( 20-25 nT) near the noon meridian (Figure 10). Hence, with increasing latitude the $B_{z}$ modulus increases; and with increasing longitude, decreases. In general, the $B_{z}$ modulus of the maximum MF strength in MC increases with increasing northern and southern minimum latitudes of the extended source. For the northern hemisphere, the relationships are more pronounced than for the southern one.

We consider the orientation of the recorded $\mathrm{MC}$ in a cylindrical model represented by a latitude angle $\varepsilon$ and a longitude (azimuth) angle $\beta$. Below are the results of the study of regularities of MC orientation, defined by solar source parameters.

\subsection{Latitudinal angle of MF vector in MC}

Distributions of the latitudinal angle of the MF vector in $\mathrm{MC}$ depending on the minimum latitude of the exten-

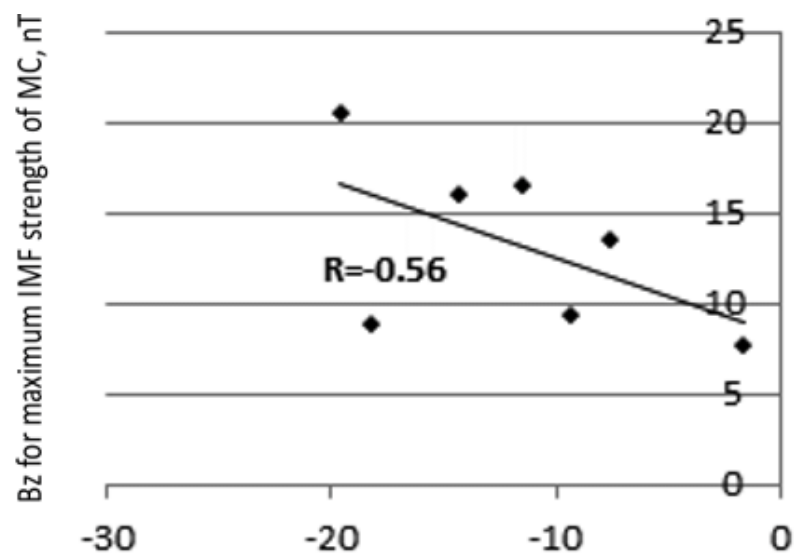

minimum source latitude, degrees

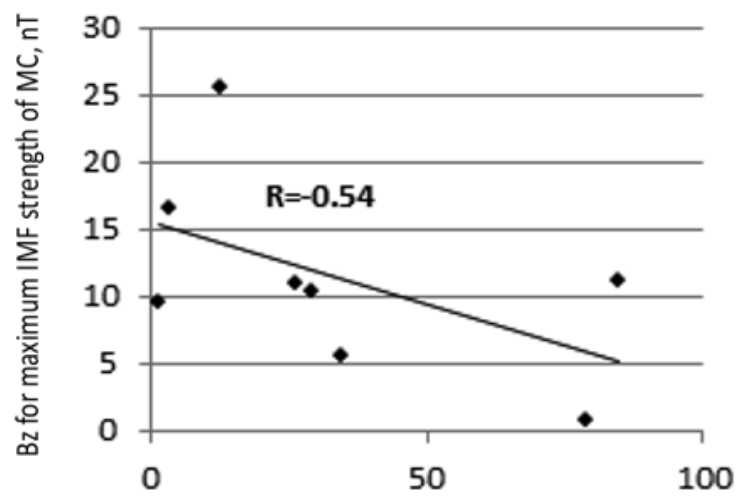

longitude corresponding to the minimum source latitude, degrees

Figure 10. $B_{z}$ component of maximum MF strength in MC versus the minimum latitude of the source $(a)$ and its associated longitude $(b)$ in the southern hemisphere

ded solar source and its associated longitude exhibit minimum modulus of the latitudinal angle of the MF vector in MC near the heliographic equator (Figure 11, $a$ ) and maximum one (up to $\sim 60-90^{\circ}$ ) near the noon meridian (Figure 11,b). With increasing solar latitude of the source, the latitudinal angle increases; and with increasing solar longitude, decreases. For the northern hemisphere (northeast and northwest), these relationships are more pronounced than for the southern one. This feature in the behavior of the latitude angle and, consequently, of the polar angle may be indicative of the most likely formation of "lying" clouds by sources located at lower latitudes (near the solar equator) [Barkhatov, Kalinina, 2010].

\subsection{Longitudinal (azimuthal) angle of $\mathrm{MF}$ vector in MC}

Distributions of the longitudinal angle of the MF vector in MC depending on the minimum latitude of the extended solar source and its associated longitude exhibit the maximum modulus of the longitudinal angle of the MF vector in MC near the heliographic equator (Figure 12, $a$ ) and near the noon meridian (Figure 12,b). It is noticeable that with increasing solar latitude and longitude the longitudinal angle decreases. For the northern hemisphere (northeast and northwest), the dependences are more pronounced than for the southern one. 


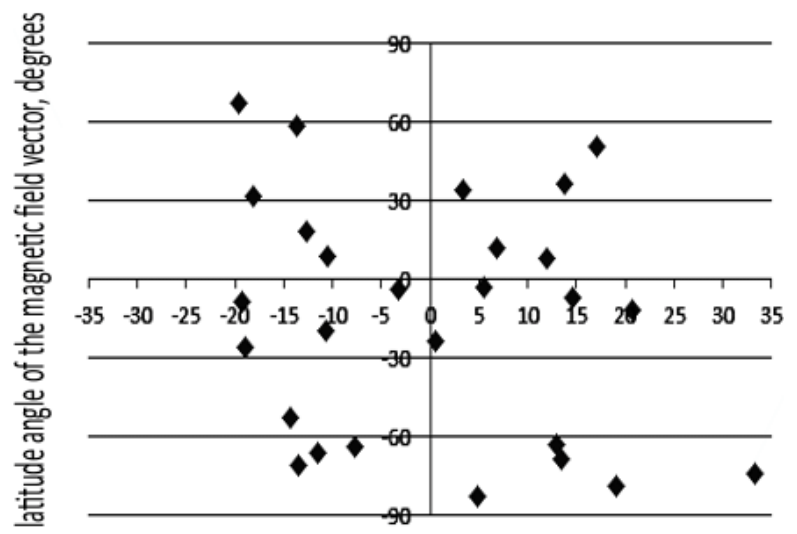

minimum source latitude, degrees

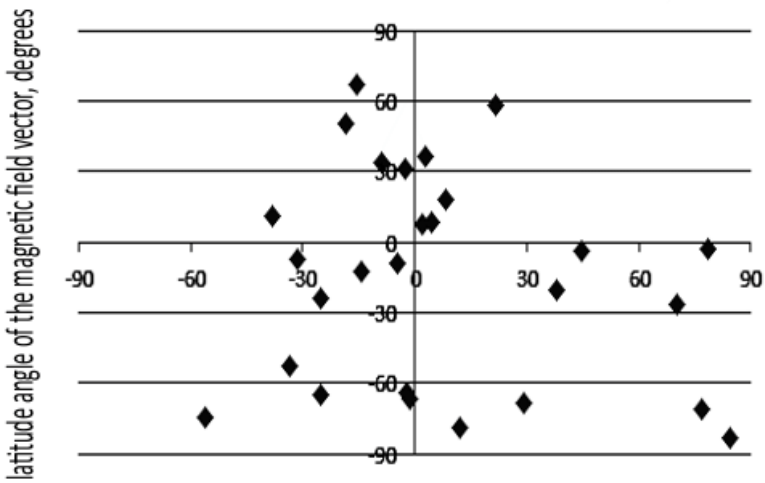

longitude corresponding to the minimum source latitude, degrees

Figure 11. Distribution of the latitudinal angle of MF vector depending on the minimum latitude of the source $(a)$ and on the longitude corresponding to this latitude $(b)$

3. ANALYZING THE RELATIONSHIP OF PARAMETERS OF CME SOLAR SOURCES WITH GEOMAGNETIC ACTIVITY OF MAGNETOSPHERIC SUBSTORMS

$\mathrm{CME}$, as a manifestation of solar activity, when impacting Earth's magnetosphere causes a global geomagnetic storm accompanied by isolated magnetospheric substorms or series of substorms. It seems, therefore, appropriate to relate the parameters of solar sources to the intensity of magnetospheric substorms, which we characterize as an enhancement of the westward electrojet, described by the $A L$ index.

3.1. Relationships of $A L$ extremum with the minimum solar source latitude and to its associated longitude taking place when the MC body interacts with Earth's magnetosphere

The relationship between the $A L$ extremum and the minimum source latitude shows that the observed $A L$ extrema increase as the solar source approaches the equator (Figure 13). It is characterized by a correlation coefficient of $\sim-0.6$ mainly for the southern solar hemisphere with a minimum in the vicinity of the heliographic equator and by lack of substorms related to the sources with minimum latitudes greater than $20^{\circ}$. For the northwest this relationship $(R \sim-0.6)$ is also observed with increasing extrema when approaching the equator, i.e. with decreasing

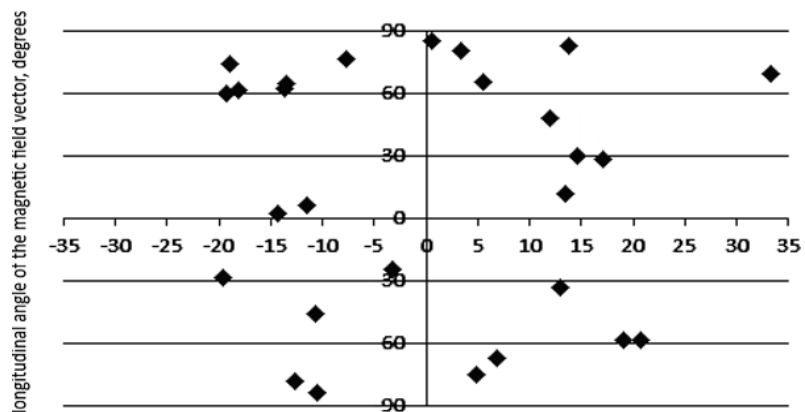

minimum source latitude, degrees

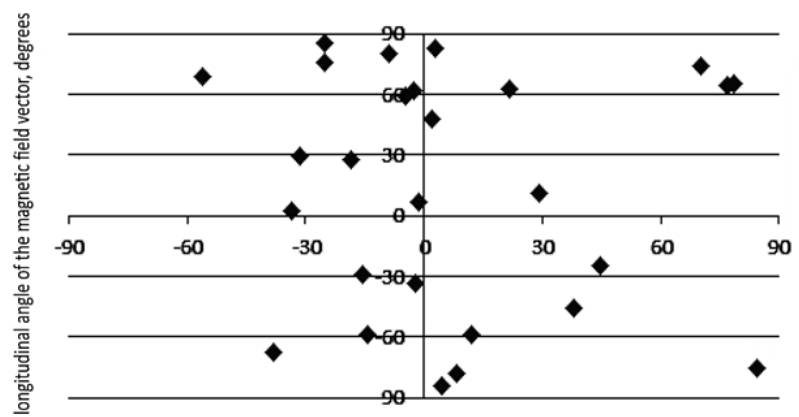

longitude corresponding to the minimum source latitude, degrees

Figure 12. Distribution of the longitudinal angle of MF vector depending on the source latitude $(a)$ and the longitude corresponding to the minimum latitude of the source $(b)$

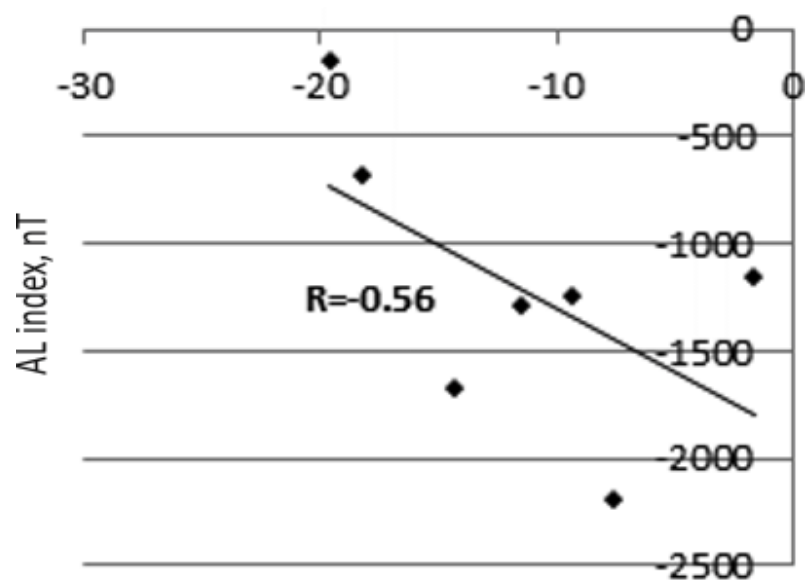

minimum source latitude, degrees

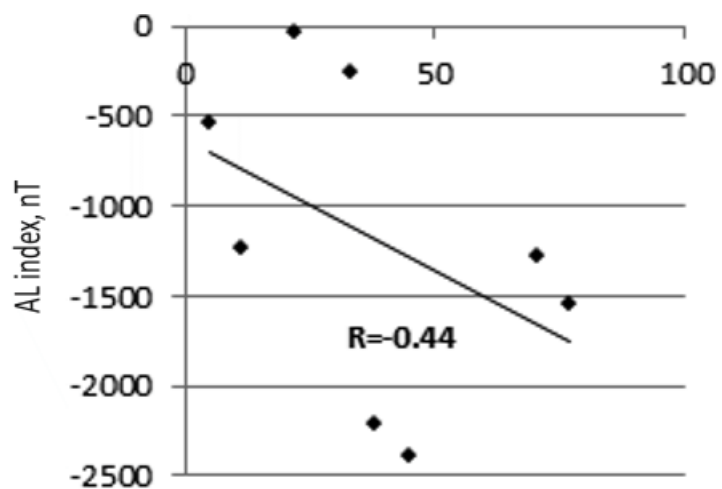

longitude corresponding to the minimum source latitude, degrees

Figure 13. $A L$ extremum versus the minimum latitude of the solar source (a) and $A L$ versus the longitude of the solar source corresponding to the minimum latitude of the source during propagation of MC body $(b)$ 
latitudes. This relationship, however, changes for the northeast, transforming into a weak direct relationship.

\subsection{Relationship of $A L$ extremum with mini- mum latitude and its associated longitude during propagation of MC turbulent sheath}

For sources in the southern hemisphere the $A L$ extremum depends on the minimum latitude with a correlation coefficient of -0.5 . Northern latitudes may feature a weak negative relationship (Figure 14).

For the northeast there is almost no negative relationship between $A L$ and longitude. For the northwest, the direct relationship is more pronounced ( 0.5). For the western hemisphere as a whole there is a weak direct relationship.

The comparison between the results of Sections 2 and 3 suggests that the substorm effectivenesses of clouds and their sheaths are comparable and determined by the location of solar sources in a similar way.

\section{CONCLUSIONS}

We have carried out a statistical study of relationships of MC-type CME parameters and geomagnetic activity level with parameters of localization of their sources on the solar disk. We have solved the main problems of identification of CME/MC sources and transfer of respective structures in the solar wind.

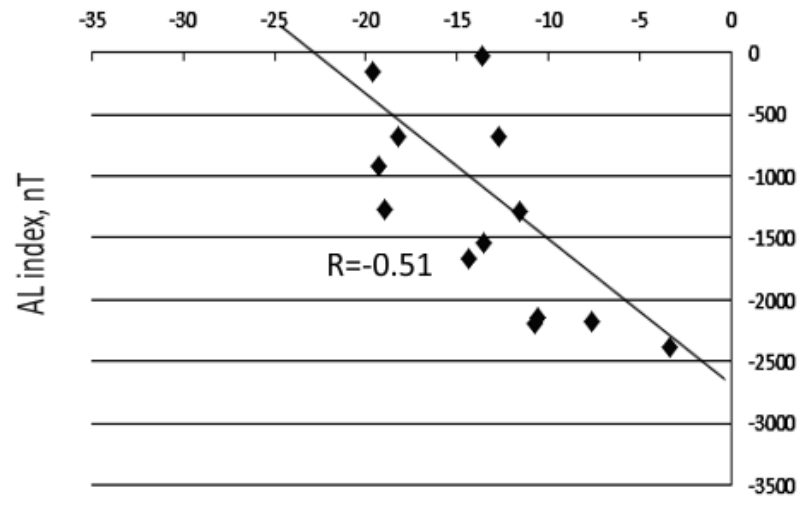

minimum source latitude, degrees

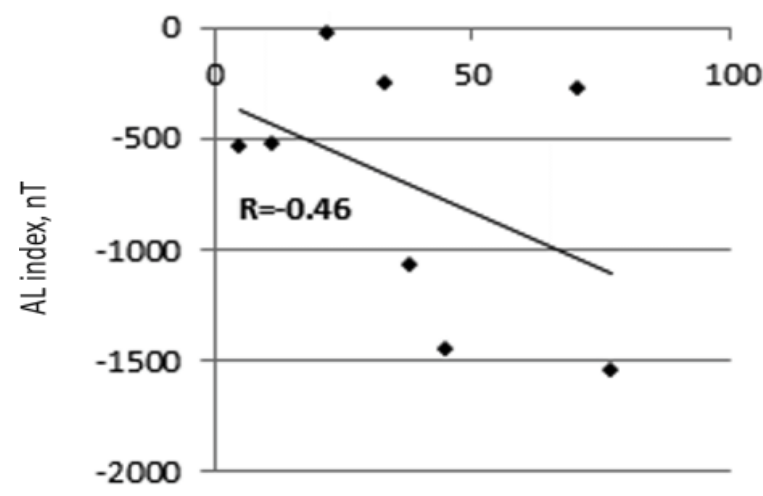

longitude corresponding to the minimum source latitude, degrees

Figure 14. $A L$ extremum versus the minimum latitude of the solar source $(a)$ and $A L$ versus the longitude of the solar source corresponding to the minimum latitude of the source during propagation of TS in $\mathrm{MC}(b)$
1. CME sources often do not stand out in the corona or photosphere, but in this case they can be determined from residual effects.

2. The isolated MC is an extremely rare phenomenon and its source difficult to detect against the perturbed solar corona or photosphere may be identified by observing the CME body as a diffuse cloud carried away from the Sun together with the frozen-in coronal magnetic field during eruption. CME bodies are readily observed by coronagraphs, and this allows us to determine their source on the solar disk and then easily identify it with the aid of patrol SC. Such CMEs also involve MCs so the MC source can be determined as well.

3. Direct propagation of isolated MCs to Earth is recorded by coronagraphs only in profile.

4. The huge size of MC allows us to derive parameters of the structure and its dynamics only on the crosssection of the object from patrol SC data. Since for the clouds we have adopted the cylindrical force-free model successfully applied in many studies, we interpolate the dynamics of the parameters on the cross-section of the complex huge pseudocylindrical structure into the entire cloud.

We have studied peculiarities of distribution of solar sources with different lengths and angular orientation of their visible part depending on source coordinates. We have established that the extended sources having a small inclination to the solar equator and located on the solar limb as opposed to nonextended and strongly inclined ones can generate expanding clouds, which most likely can reach the magnetosphere, as well as clouds from a source near the zero meridian and low latitudes.

We have established regularities of relationships of magnetic and orientation characteristics of MCs recorded by SC with localization and spatial parameters of their sources on the solar disk. It should be noted that in some cases the relationships considered in certain quadrants of the solar disk are absent or not established. This is not surprising since the exact identification of solar activity sources with $\mathrm{MC}$ structures propagating through patrol SC is difficult for the reasons mentioned in Introduction. Nevertheless, we have examined the following relationships: between $\mathrm{MC}$ and the minimum latitude of the source and its associated longitude; between the maximum magnetic field strength in $\mathrm{MC}$ and the minimum latitude of the source and its associated longitude; between $B_{z}$ modulus of the maximum MF strength in $\mathrm{MC}$ and the minimum latitude of the source and its associated longitude; between latitudinal and longitudinal angles in the solar-ecliptic coordinate system for the MF vector in $\mathrm{MC}$ and the minimum latitude of the source and its associated longitude; we have determined their characteristic features (see Sections 2 and 3). There is also an increase in the number of high-speed MCs related to the eastern solar disk and in the number of slow MCs related to the western one, which reflects effects of the spiral motion of the solar wind stream. We have found that in a large group of MCs their maximum magnetic field depends on source coordinates. The maximum MF strength in MC increases with northern and southern latitudes, starting from the equator, which is consistent with the most likely distribution of magneto- 
active regions over the solar disk. The same is true for the MF $B_{z}$ component.

Analysis of orientation of magnetic clouds, considered in the cylindrical model, has demonstrated the most probable formation of clouds lying near the plane of the ecliptic by low-latitude sources.

We have also examined the relationship between substorm activity (according to the $A L$ index) and parameters of MC solar sources during periods when Earth's magnetosphere is affected by MC structures (body and sheath). We have found that there are no substorms associated with extended sources outside the heliolatitude range $\sim 5-20^{\circ}$. Thus, we have established relationships between solar source coordinates and geomagnetic activity of both the MC sheath and body. They are consistent with the most probable distribution of magnetoactive regions over the solar disk.

This work was funded by RFBR grant No. 18-3500430 (Barkhatova O.M., Revunova E.A., Romanov R.V.) and State Task of the Ministry of Education and Science of the Russian Federation No. 5.5898.2017/8.9 (Barkhatov N.A., Revunov S.E.).

\section{REFERENCES}

Barkhatov N.A., Kalinina E.A. Determination of magnetic cloud parameters and prediction of magnetic storm intensity. Geomagnetism and Aeronomy. 2010, vol. 50, no. 4, pp. 453460. DOI: $10.1134 /$ S0016793210040043.

Barkhatov N.A., Kalinina E.A., Levitin A.E. Manifestation of configurations of magnetic clouds of the solar wind in geomagnetic activity. Cosmic Res. 2009, vol. 47, no. 4, pp. 268-278.

Barkhatov N.A., Revunova E.A., Vinogradov A.B. Effect of orientation of the solar wind magnetic clouds on the seasonal variation of geomagnetic activity. Cosmic Res. 2014a vol. 52, no. 4, pp. 269-277. DOI: 10.1134/ S0010952514040017.

Barkhatov N.A., Revunova E.A., Levitin A.E. Classification of space-weather complexes based on solar source type, characteristics of plasma flow, and geomagnetic perturbation induced by it. Geomagnetism and Aeronomy. 2014b, vol. 54, no. 2, pp. 173-179. DOI: 10.1134/S0016793214020030.

Barkhatov N.A., Vorobjev V.G., Revunov S.E., Yagodkina O.I. Effect of solar dynamics parameters on the formation of substorm activity. Geomagnetism and Aeronomy. 2017, vol 57, iss. 3, pp. 251-256. DOI: 10.1134/S0016793217030021.

Barkhatov N.A., Revunov S.E., Vorobjev V.G., Yagodkina O.I. Studying the relationship between high-latitude geomagnetic activity and parameters of interplanetary magnetic clouds with the use of artificial neural networks. Geomag netism and Aeronomy. 2018, vol. 58, iss. 2, pp. 147-153. DOI: 10.1134/S0016793218020020.

Barkhatova O.M., Kosolapova N.V., Barkhatov N.A., Revunov S.E. Synchronization of geomagnetic and ionospheric disturbances over Kazan station. Solar-Terrestrial Physics. 2017, vol. 3, iss. 4, pp. 58-66. DOI: $10.12737 /$ stp-34201706.

Burlaga L.F., Wang C., Richardson J.D., Ness N.F. Evolution of the multiscale statistical properties of corotating streams from 1 to 95 AU. J. Geophys. Res. 2003, vol. 108, no. A7, pp. 1305-1310. DOI: 10.1029/2003JA009841.

Hundhausen A.J. Coronal mass ejections. The many faces of the Sun: A summary of the results from NASA's Solar maximum mission. N.Y. Springer, 1999. 143 p.
Ivanov K.G. Solar sources of interplanetary plasma flows in the Earth's orbit. Geomagnetism and Aeronomy. 1996, vol. 36, pp. 19. (In Russian).

Kilpua E.K.J., Lee C.O., Luhmann J.G., Li Y. Interplanetary coronal mass ejections in the near-Earth solar wind during the minimum periods following solar cycles 22 and 23 . Ann. Geophys. 2011, vol. 29, pp. 1455-1467. DOI: 10.5194/angeo29-1455-2011.

Kilpua E.K.J., Li Y., Luhmann J.G., Jian L.K., Russell C.T. On the relationship between magnetic cloud field polarity and geoeffectiveness. Ann. Geophys. 2012, vol. 30, pp. 10371050. DOI: 10.5194/angeo-30-1037-2012.

Liu Y., Manchester IV W.B., Richardson J.D., Luhmann J.G., Lin R.P., Bale S.D. Deflection flows ahead of ICMEs as an indicator of curvature and geoeffectiveness. J. Geophys. Res. 2008, vol. 113, iss. A9, CiteID A00B03. DOI: 10.1029/2007JA012996.

Manakova Yu.V., Pekhteleva K.A., Barkhatov N.A., Revunov S.E. Space-time analysis of disturbances in the PC4-5 period range during magnetic storms by correlationskeleton method. Vestnik Miniskogo universiteta [Vestnik of Minin University]. 2016, no. 1, pp. 1-6. (In Russian).

Neugebauer M., Liewer P.C. Creation and destruction of transitory coronal holes and their fast solar wind streams. $J$. Geophys. Res. 2003, vol. 108, no. A1, pp. 1013-1016. DOI: 10.1029/2002JA009326.

Nikolaeva N.S., Yermolaev Y.I., Lodkina I.G. Dependence of geomagnetic activity during magnetic storms on the solar wind parameters for different types of streams. Geomagnetism and Aeronomy. 2011, vol. 51, no. 1, pp. 49-65. DOI: 10.1134/S0016793211010099.

Riazantseva M.O., Dalin P.A., Zastenker G.N., Parhomov V.A., Eselevich V.G., Eselevich M.V., Richardson J. Properties of sharp and large changes in the ion flux (density) of the solar wind. Cosmic Res. 2003, vol. 41, no. 4, pp. 371-381.

Wang Yuming, Chen Caixia, Gui Bin, Shen Chenglong, Ye Pinzhong, Wang S. Statistical study of coronal mass ejection source locations: Understanding CMEs viewed in coronagraphs. J. Geophys. Res. 2011, vol. 116, iss. A4, CiteID A04104. DOI: 10.1029/2010JA016101.

Wu C. C., Lepping R. P. Effects of magnetic clouds on the occurrence of geomagnetic storms: The first 4 years of Wind. J. Geophys. Res. 2002, vol. 107, no. A10, pp. 1314-1321. DOI: 10.1029/2001JA000161.

URL: $\quad$ http://lasco-www.nrl.na-vy.mil/index.php?p=content/cmelist (accessed May 6, 2019).

URL: https://cdaw.gsfc.nasa.gov/CME_list/ (accessed May 6, 2019).

URL: http://umtof.umd.edu/sem/ (accessed May 6, 2019).

URL: ftp://ftp.ngdc.noaa.gov/STP/SOLAR_DATA/ (accessed May 6, 2019). 2019).

URL: http://vso.nso.edu/cgi/catalogue (accessed May 6,

How to cite this article

Barkhatov N.A., Revunova E.A., Barkhatova O.M., Romanov R.V., Revunov S.E. Studying the relationship of localization parameters of solar sources of magnetic clouds with their characteristics and substorm activity. Solar-Terrestrial Physics. 2019. Vol. 5. Iss. 3. P. 59-67. DOI: $10.12737 /$ stp-53201907. 